\title{
Digital Practices \& Applications in a Covid-19 Culture
}

\author{
Christina Romero-Ivanova ${ }^{1}$, Michael Shaughnessy ${ }^{2}$, Laura Otto ${ }^{3}$, Emily Taylor ${ }^{3} \&$ Emma Watson $^{3}$ \\ ${ }^{1}$ Assistant Professor of Education, Indiana University Kokomo, USA \\ ${ }^{2}$ Professor of Special Education, Eastern New Mexico University, USA \\ ${ }^{3}$ Undergraduate students, Indiana University Kokomo, USA \\ Correspondence: Christina Romero-Ivanova, Indiana University Kokomo, USA. E-mail: civanova@iuk.edu
}

Received: July 2, 2020

doi:10.5539/hes.v10n3p80
Accepted: July 18, $2020 \quad$ Online Published: July 24, 2020

URL: https://doi.org/10.5539/hes.v10n3p80

\begin{abstract}
This article addresses reflections of one University instructor's teaching and her pre-teacher education students' innovative digital learning practices during the Covid-19 pandemic in Spring 2020. The question of How has one instructor embedded digital practices in her virtual teaching to engage and purposefully introduce and connect pre-teacher education students with diverse technologies and multimodalities of learning during a mandatory virtual instruction time? will be addressed and discussed.

Student-centered practices such as group work, pair work, the use of Zoom breakout rooms, and multimodal literary responses through technology applications such as Flipgrid and Google Docs will be described and reflected upon. The instructor's own teaching practices that have included weekly mentoring meetings with her education students and continuing individual coffee meetings in diverse settings will be highlighted as ways of demonstrating care and encouragement toward face-to-face students who have been transitioned as online students. The reflections outlined in this abstract draw upon the notion of technologies as providers of active interactions and will include snapshots of an instructors' students' digital artifacts such as Flipgrid, video-recorded monologues, and Google Doc news stories with students reflecting on the uses of multimodal technologies in their own future teaching practices. This manuscript will also include student reflections and a sidebar of suggestions for using Zoom with virtual teaching.
\end{abstract}

Keywords: digital literacies, Flipgrid, Google Docs, teacher education, Zoom

\section{Introduction}

The crisis started on the campus of the University of Washington, as students, faculty and administration gradually became aware of a pending crisis, which became an epidemic, and then slowly morphed into a pandemic. It was first known as a coronavirus, then Covid-19, and then massive communication from the White House began to explain to the American public the seriousness of the situation. Gradually, colleges, universities, and community colleges were faced with several challenges. While some courses were already offered online, there were a large number of courses that were "in person" and these courses had to be quickly, almost overnight morphed into some version of an online class-utilizing whatever technologies that faculty were familiar with at the time. Some courses such as music, art, dance, theatre, drama, physical education, and science courses involving labs faced major difficulties. Music students could not perform their senior recitals, and private lessons became impossible. Some instructional technologists moved quickly to improvise, and other faculty simply assigned additional work to be completed and submitted via e-mail attachment. Other faculty employed Media Site and Microsoft Teams (Barry and Kanematsu, 2020) and almost overnight Zoom became a device that was purchased by faculty out of their own funds to complete the semester with at least a semblance of academic integrity and fidelity. Master's theses had to be completed at a mutually convenient time and schedules had to be juggled to assist students to graduate on time.

Students at the same time encountered stress, financial, and emotional difficulties. Foreign students were unsure about travel arrangements and airports began to close. Add to this the very real logistical difficulties of closing dorm rooms and transporting one's possessions. Truly a crisis of extreme magnitude was confronting all educational institutions, not just across America, but literally the world. 


\section{Background}

During the spring 2020 semester, Covid-19 created new norms and shifted individuals' lives into a "new normal". Affected by these shifts were higher education institutions with instructors and students moving to online synchronous and asynchronous instruction and digital practices. This article discusses the overarching nature of digital applications and practices as a segue into one instructor's digital teaching and her students' digital learning and responsive practices during the Covid-19 pandemic. Most importantly, some students shared their reflections from having experienced online and synchronous learning in one course "Using Computers in Education" during the pandemic. They share their experiences with using Zoom for synchronous instruction and reflect on activities that involved Flipgrid, Google Docs, and Razkids.com as differentiated ways of responding.

As Greenhow, Robelia, and Hughes (2009) remark, $21^{\text {st }}$ century technologies have promoted differentiated ways of teaching and learning that are highly interactive.

The meaning or purpose of this study was to immediately explore and examine the crisis confronting both instructors and students during the Covid-19 crisis. Though this is not an empirical study, but a very preliminary exploratory study, it offers a testimony to the power of connectedness. Digital practices that we have engaged in, and ones we discuss in this article, along with the power of the human spirit of collaboration speak to the ability to overcome and to continue in purposeful ways.

\section{Literature Review}

Much has been written about instructional technology and the discipline of online teaching (Shaughnessy and Fulgham, 2011). Indeed, over the past 20 years many worthy books have been written and cited and many journals have developed to address the issue of online learning. Shaughnessy, Johnson, Flores, and Singh (2019) have most recently examined the factors that contribute to student success- in both online and media site courses. Faculty continue to need much assistance in the use of LMS (Learning Management Systems) in terms of utilizing feedback and the deployment of both visual modalities and auditory modalities. Instructors are not often aware of the importance of feedback as well as the importance of almost instantaneous feedback.

There was a quite novel approach to providing feedback and allowing faculty to comment on their concerns regarding teaching during a time of pandemic. Several scholars were asked to share their thoughts, feelings, and concerns regarding how the Covid-19 even impacted their classes, their instruction, and their students. Martin, et al (2020) in Places Journal gave their own singular perspectives about what they referred to as :"emergency online instruction" Each one of these scholars share their perceptions and reflections as to their challenges and the impact on their instruction and most importantly student learning. In this article, Brand offers the most poignant comment: "I miss my students. I sense they are hurting. I see that they are stressed and the distance makes it harder to know what I can do to help or how I can help them find a respite amidst all the anxiety that is outside their door, and sometimes inside their space".

Sturlaugson explored and examined the issue of evaluation of student work: "And how do I evaluate the work coming from a student whose personal space is layered with uncontrollable distractions, evident every time they unmute to participate in a discussion?"

Some faculty simply recognized these "extenuating circumstances" and were liberal in their grading criteria and others provided additional assistance, and extended time frames and accommodations for those students whose lives were disrupted by this event.

Isenstadt has indicated that faculty were "at the same time trying to assess the various options, such as voiceover power points, Zoom meetings, Panopto. etc " and indicated that this was the most pressing challenge for faculty.

It should be noted that a series of these articles then appeared, with a wide variety of other scholars offering their insights and robust reflections. This particular series of reflections brought home the very wide number of concerns that faculty have in the short run and in the long run-not just in terms of impact on students and faculty research - but on their respective fields and society at large.

There were several follow-up installments featuring scholars from all over the world, who were attempting to cope with the pandemic and continue to provide adequate support, instruction, and mentoring for their students.

Barry and Kanematsu (2020) reviewed some of the issues and concerns about having to almost overnight transition from face to face to online instruction. The authors, one from New York and one from Japan, described their use of Zoom, and its capacity to mute in order to expedite clarity, and visual screens so as to foster and nurture face to face contact and interaction. Citing the work of Bellan (2020) they elaborated on its use in their respective engineering realms. There are certainly positive and negative aspects but Zoom appeared to be almost a magic solution to the 
Covid-19 pandemic. They further discussed the use of avatars, gamification and Second Life and discussed the pros and cons of these supplementary approaches. Some students were aware of Second Life and were able to navigate it fluidly, whereas others had some difficulty as they had not prior knowledge or experience All of these approaches incorporate a certain degree of novelty and a visual approach to learning.

Cuellar (2002) extensively wrote about the challenges and issues surrounding the transition from in-person to online instruction, specifically for nursing students. Kebritchi, Lipschuetz, Santiague (2017) have extensively reviewed both the issues and challenges for teaching successful online courses in higher education. The main issues to be addressed in this paper is the extreme transition from classroom instruction to online instruction (using various platforms such as Blackboard, Canvas, Brightspace) and the concurrent utilization of new technologies (Zoom, Microsoft Teams, Google Classrooms, Kanopy, Media Site, and various other devices. Internet sources were scoured for relevant resources (such as YouTube) and e-mail and Zoom seemed to become the preferred modes of communication almost overnight. Burke (2020) was one of the first to write about, interview and investigate and discuss the difficulties in making a quick transition to online instruction in higher education. The terms "successful but stressful" reiterated during her article posted online in Inside Higher Ed also reflected on the dilemma that some classes simply could not be adapted, such as dance. Ebrahimji (2020) writing for $\mathrm{CNN}$ wrote about the transition from a student point of view.

Interviewing several students, many were disgruntled about not being able to complete their course work in film, dance, and math. Many were upset about paying a lot of tuition money to "sit on the floor" Cheating on assignments and tests and exams was also discussed as an issue.

ETS (2020) quite rapidly began to offer a variety of training for instructors thrust into an online environment. They offered training in a) course design b) communication and engagement c) building assessment and grading issues, d) Video Note and You Tub e) and the conducting of virtual meetings.

\subsection{Media Site Instruction}

For some instructors, they already had some Media Site Presentations on various topics pre-recorded. For other instructors, they were charged with having to get proficient at this mode of delivery almost overnight. Media site Presentations can be readily accessed by students and can serve as a supplement to the text and to whatever power points may be available. Media Site presentations enable instructors to provide an overview of a chapter and then provide individual responses to any questions via the Discussion Board.

McGuigan (2020) focused on some of the main tasks and chores that confronted faculty in terms of transitioning. These are:

- $\quad$ Learning new software

- Teaching students to use new software

- $\quad$ Providing captions and interpreters

- $\quad$ Navigating copyrights

- Helping students' cope with isolation

- Balancing our new family life and work

- Feeling disconnected from our students

In terms of learning new software, this is an issue in and of itself. For some faculty the learning curve may be quite steep, and frustration and exasperation may ensue. For other faculty who may have a student with special needs (vision, hearing) then making accommodations and modifications has become more difficult and accessibility must be addressed within a very brief time frame to serve that student. Communication Services for the Deaf is a resource that not all instructors or students with disabilities services personnel may not be aware of.

\subsection{Sensitivity and Compassion toward Students during Transitions}

Instructors were put into a quite difficult position- asking students to adjust to an entirely new mode of pedagogy- as their classes approached mid-terms. Instructors needed to employ an entirely new approach to teaching a class (for example Western Civilization) that had been taught in person in a lecture hall for several years. Students in certain domains, for example student teaching, were left without a school to go to, without students to teach, and in some cases at a distance from their student teaching supervisors. Graduation concerns became apparent as seniors were looking forward to a large ceremony and these after being cancelled were put "online" in a virtual format- although some students were allowed to "walk" at the next in-person ceremony.

Fisher \& Frey (2020) attempted to provide several steps for a rich, robust, distance learning experience. They 
emphasize making coursework predictable, structured, and the need to establish a routine with clear concise expectations. They further cite Cornelius-White (2007) who elaborated on and described some of the most critical aspects of several effective, efficient teacher-student relationships.

These include:

- Teacher empathy: understanding students' experiences, interests, values, and goals

- Unconditional positive regard: warmth in interactions and feedback

- Genuineness: the teacher's ability to be authentic and honest with students

- Non-directivity: student-initiated and student-regulated activities that are supported by the teacher

- Encouragement of critical thinking: focusing on students' thinking as opposed to traditional memory emphasis"

In a sense, teachers are almost going to need to work on all of the above, at least initially, reflecting on, and commenting on the difficulties that some students have had. Further, they should allow for catharsis, and a sharing of what has transpired. Teachers need to support time for students to "re-learn" their peers and to almost "re-learn" interpersonal relations, and in a sense to "catch up" with their friends whose lives have also been disrupted.

\subsection{Communication and Collaboration with Others}

If students were familiar with Blackboard and the Discussion Board, the transition was somewhat facile. If instructors were familiar with Blackboard and had experience with other LMS, the transition was less problematic. Students may have been able to check the Announcements Board and the Discussion Board and the Instructor could have utilized the Discussion Board as a type of support group for students to share their concerns and legitimate complaints.

The internet, while it has never been completely reliable or dependable, was found to be even more problematic during this crisis period, and globally students who lived in rural areas, ones who lived in areas that did not have adequate $\mathrm{Wi}-\mathrm{Fi}$, or students who could not afford access were often marginalized because of these issues. There were some problems with access to the internet, Zoom, and other important instructional aspects during this time, but Indiana University Kokomo, as other institutions, was able to provide drive-up Wi-Fi access for its students. Students could come to campus and park in the main parking lot to receive Wi-Fi. Universities also offered extended hours in which students could utilize computer stations, conduct research, and print documents.

\subsubsection{Zoom Breakout Rooms}

Zoom breakout rooms enabled faculty to establish first a communication with students who did not respond to emails and secondly, a visual face to face communication to ensure understanding of the course content and materials. A split screen is available for instructors to use, and instructors can "mute" and unmute students and to prompt via Chat for their input and feedback.

\subsubsection{Face Time}

Just as Zoom allowed faculty to communicate with students virtually and face-to-face, FaceTime allowed for synchronous communication at a moment's notice. Students and instructors could conveniently chat with one another from different locations about issues with assignments, questions about projects, or to just check in.

\subsubsection{Kaltura}

The challenge of quickly transitioning to an online teaching/learning environment caused shifts in schedules and in many ways, a restructuring of lives. Sometimes, Zoom instruction times and students' work and course schedules misaligned. Kaltura, as a media space that offers the ability to record Zoom sessions, offered absent students the ability to view recorded sessions on their own time. This media space, which allows for asynchronous viewing provided an equitable learning space so that students could remain engaged and in sync with their class.

\subsubsection{Google Docs}

As a synchronous writing application, Google Docs offered students a collaborative space that was accessible and practical. In this article, students describe their use of Google Docs in regards to their assignment of creating a news story related to our reading of scenes from A Midsummer Night's Dream. Besides being an application, which students found they could easily understand and use, it also pressed them to creatively expand their knowledge on characters' thoughts and motives and challenged them to create rather than to only take in 
knowledge.

\subsubsection{Flipgrid}

Flipgrid is an online application which allows contributors opportunities to respond in different ways by providing a space in which they can upload videos, submit text, or create and post presentation slides. Spring 2020 semester called for differentiation already, in the process of instruction, in the process of learning, in the process of communicating with others, and so using Flipgrid was an extension of what many were already doing in the classroom. Students' reflections in the next sections note the ways in which Flipgrid and differentiated instruction and learning helped them to consider practices they would enact in their own future classrooms with students.

As instructors, we (Christina and Michael) were aware of the concerns regarding security of Zoom data, for example, but under the exigent circumstances we were forced to proceed with caution. The pandemic situation had already established isolations in which students were not able to socialize, attend school face-to-face, and to connect with others in greater degrees. Zoom allowed for connections that would not have been possible through the sole uses of texting, emailing, or online coursework. Regarding data concerns, we were able to establish zoom waiting rooms, passwords to validate users, and admitted students individually into Zoom sessions to protect privacy and the sanctity of the learning environment.

\subsection{A Range of Emotions}

Cut off from their peer group, from their support system, from their teammates and the camaraderie that is often provided on a collegiate team, student athletes saw their season cut short. For seniors, playing their last few collegiate games, this was an extremely difficult emotional thing to address and process. Relationships were ripped asunder. Marriages were delayed or postponed. And for some students, their part time employment was terminated. For some students, clinical depression did set in. For others, this was equivalent to somewhat of a post-traumatic stress event. Needless to say, with air travel minimized, students had to make some very quick decisions as to where to live, and with the school cafeteria closed- where to eat. The entire event was and is, daunting and anxiety provoking as students raised concerns about their grades and GPAs. And these are legitimate claims.

In terms of students and teachers returning to the classroom, it has been indicated that instructors need to show "sincere care" and recognize and validate the extremely stressful experiences that they have encountered. Some students have had to withdraw from classes, some have missed graduation ceremonies, some have had relationships end, and some have seen their off campus employment end. Still others have just had their entire lives disrupted. Their plans to graduate in a timely fashion have gone astray and they have had to re-organize and readjust to a new world where people are walking around with face masks and it is difficult to ascertain their emotional states (Welborn and Gringle, 2020).

Welborn and Gringle, (2020) suggest that all instructors be proactive- this has been a difficult few months and it is going to take a few months to recover to the new normal as they say. Instructors can attempt to involve pupils directly- perhaps asking them to discuss or post or email some of their concerns. There should be an attempt to decrease formality because in a sense, "we are all in this together" and we have all gotten through this togetherand are still getting through it in some instances. There should be an acknowledgement of the stress, the strife, and the struggles that we have all gone through over the past few weeks. Compassion, care, concern should be offered, and students should be welcomed back after a quite difficult time.

\subsection{The Learning Curve}

Although it has been mentioned in the literature, the idea or construct of "the learning curve" became increasingly relevant in this crisis. Faculty, and in many instances, students had to transition and learn how to teach and learn in an online environment virtually overnight. Reactions from students were mixed. Indeed, one student confessed to one author that they had never done a PowerPoint presentation and were never trained in how to do a Prezi or PowerPoint. Another student was attempting to complete a class on their cell phone to their exasperation, frustration, and concern about their grade. The uses of different technology applications in one class, "Using Computers in Education" engaged students and allowed them the freedom to purposefully work with one another. These applications and their uses from students' perspectives are described in the following section. 


\section{Reflections from the Classroom}

\subsection{Zooming Together}

\subsubsection{Laura's Experience}

Being heavily involved with classroom discussions and active participation in the new Covid-19 teaching/learning culture meant a change in practice and pattern, and Zoom breakout rooms helped us to smoothly transition. Though transitioning to online learning was going to feel differently and reposition our classroom pattern of behaviors, this new process of learning became more fluidly accomplished with the use of Zoom breakout rooms. Using breakout rooms allowed me to feel connected and aided me in remaining academically focused to finish the semester successfully. Honestly, there were times when motivation was lacking, and Zoom, along with professors' diligence helped to keep us on track. This different way of learning was challenging, but our classroom connections with one another remained intact.

With Zoom, lessons were carried out relatively smoothly with only minor tweaks. One of the first projects for our Using Computers in Education class that was to be completed during the quarantine was creating a classroom website. With the help of Zoom, our professor was able to go through each step of the process, which helped immensely. At first it seemed to be a challenge but the use of Zoom breakout rooms created collaborative workspaces just as if we had been on campus. It allowed for my group to spend time on our project during class, which was helpful. Having Zoom during this time helped more than just for successful completion of assignments and projects. It helped me to stay connected to my classmates.

\subsubsection{Emma's Experience}

The Covid-19 global pandemic forced millions of people to remain in their homes for months on end. The quarantine also made contact with others outside of the home challenging. The use of Zoom breakout rooms in college classes greatly helped to curve the negative feelings brought on from quarantining at home. Because Zoom offers breakout rooms as differentiated spaces for collaboration, it allowed my professors to place us together in separate video chats. In the breakout rooms we were able to work on group projects, study together, and talk about our personal lives. Working in Zoom breakout rooms, as extensions of our physical classroom spaces became very beneficial for all of us. These allowed for more natural ways of communicating with classmates. Video calls made possible through such applications as Zoom provided more authentic connections than text messaging or emails. Along with calming the anxiety felt during isolation, Zoom aided with group projects' efficiency. Text messages and emails can often take time to receive responses, but breakout rooms allowed for immediate communication, which resulted in my group swiftly finishing our assignments. Overall, without the use of Zoom breakout rooms I would have struggled during the time of uncertainty.

\subsubsection{Emily's Experience}

Zoom became vital in many individuals' lives to maintain jobs, preserve relationships, or as in my case, to continue education. Zoom became the mediator to many activities during this time of isolation. When not in person, my professors were able to creatively adapt their lesson plans to the circumstances. The utilization of breakout rooms allowed us to feel the personal connections we had missed with our peers. There is always a different dynamic when the instructor releases responsibility onto a group, and we all were lacking this small group insight in our new normal of virtual learning. However, breakout rooms allowed for reflection and team-building for assignments. For example, one instructor used the breakout rooms in almost every lecture to either allow us time to collaborate on an assignment or reflect on soon approaching projects. For some students, including me, it was the only time we were able to collaborate because of differing work schedules, class times, and other life challenges. The breakout rooms created a sense of connectivity to my peers that I had lost due to the mandated isolation. I was excited to get ready for the Zoom meetings because I was eager to see my friends and instructors in person. Zoom broke up the monotony of the same four walls everyone was getting to know so well and gave us all purpose to waking up and getting ready even amongst a time of stress and anxiety. Zoom meetings embraced the feeling of a classroom setting we had prior to the Covid-19 pandemic.

\subsection{Using Google Docs}

\subsubsection{Emma's Experience}

One of our class assignments for Using Computers in Education was to use Google Docs to create a news article about characters' experiences from Shakespeare's A Midsummer Night's Dream (see Fig. 1 below). I was placed in a group with three other classmates, in which we began collaboration through text messaging to manage contact and delegate responsibilities. 

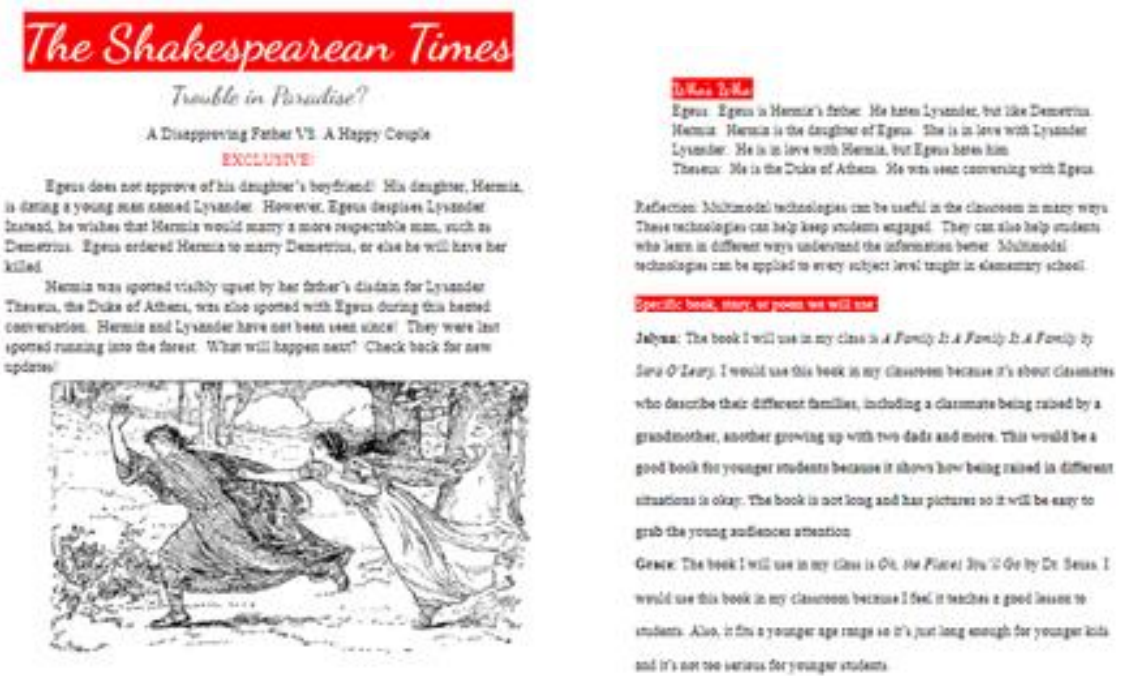

Figure 1. Trouble in Paradise? Emma's group's News Story

Using Google Docs for this project was extremely helpful. Google Docs allowed us to work steadily at our own pace, while managing day-to-day activities. Because Google Docs allows collaborators to work simultaneously, we were each able to flexibly contribute to the writing. In my experiences, other technology applications are a hassle to use because they require one collaborator to finish before sending to another. Google Docs is amazing in the sense that it allows for synchronous collaboration.

\subsection{Flipgridding Shakespeare}

\subsubsection{Laura's Experience}

Flipgrid was a very new technology to me. Having used Google Docs and Zoom, I was already familiar with some technology applications, but Flipgrid was one that was unfamiliar. Our first assignment with Flipgrid in our course Using Computers in Education involved providing a group response for how to utilize digital stories for English Language Learners. My pair partner and I were able to video record our response so that the instructor and others could easily access this. I really enjoyed the differentiated easy that Flipgrid allows. It provides a space in which to connect with peers and to collaborate and respond in a different way compared to just writing a paper. Flipgrid also allowed us to explain knowledge we had gained, enacting roles as teachers.

Using Flipgrid allowed for understanding a differentiated way for students to richly respond. It was a neat experience and something that was engaging. The videos I created, I keep and plan on using someday for my own classroom.

\section{Conclusion}

This paper has attempted to cursorily review the recent Covid-19 dilemma and the challenges, stresses and difficulties, it has presented for students, faculty and even parents who have had to assume instruction for their children who were no longer attending school but were receiving instruction via the Internet. It has also provided reflective evidence of student engagement through the uses of different kinds of technologies and applications during the Covid-19 time of online education in one pre-teacher education course. The intent of this paper was not to prove or disprove any hypothesis but rather to take a preliminary examination with a small sample size, thus generalizability is limited.

\section{Implications for Classroom Use}

This pandemic time has allowed us to see just how much education can be modified for the ability to learn anytime and anywhere. Our hope, as university professors and future teachers, is that life will return to normal and that we are able to physically teach in classrooms filled with students. However, the ability to virtually teach and learn has been an enriching experience that has taught preparedness for future instruction in our classrooms and have caused a heightened awareness of different technologies and teaching methods that can be used to engage learners.

In the past we have experienced such calamities as the floods in New Orleans and 9/11. Teachers, instructors, and students need to be prepared for various major disruptions and to be able to utilize and even reinvent their 
ways of teaching and learning.

\section{References}

Barry, D. M., \& Kanematsu, H. (2020). Teaching during the COVID-19 Pandemic. Online Submission.

Bellan, R. (March 24, 2020). What You Need to Know about Using Zoom, Forbes. Retrieved from https://www.forbes.com/sites/rebeccabellan/2020/03/24/what-you-needto-know-about-using-zoom/\#439e80 df3284

Burke, L. (2020). The big transition. Retrieved from https://www.insidehighered.com/news/2020/03/31/faculty-discuss-their-quick-transition-online-instruction

Cuellar, N. (2002). The Transition from Classrooms to Online Teaching. Nursing Forum, 17(3), 5-13. https://doi.org/10.1111/j.1744-6198.2002.tb01005.x

Ebrahimiji, A. (2020). Students navigate uncharted territory as pandemic forces education online. Retrieved from https://www.cnn.com/2020/03/16/us/online-school-coronavirus-trnd/index.html

Educational Testing Service. (2020). Transitioning from classroom to online instruction Brightspace Trainings schedule. Retrieved from

https://sites.pstcc.edu/elearn/d21/transition-from-classroom-to-online-instruction-brightspace-trainings-sche dule/

Fisher, D., \& Frey, N. (2020). 4 Steps for Powerful Distance Learning Experiences. Retrieved from https://smartbrief.com/original/2020/07/4-steps-powerful-distance-learning-experiences

Kebritchi, M., Lipschuetz, A., \& Santiague, L. (2017). Issues and Challenges for Teaching Successful Online Courses in Higher Education: A Literature Review. Journal of Educational Technology Systems, 46(1), 4-29. https://doi.org/10.1177/0047239516661713

Martin, R., Piedmont-Palladino, S., Sturlaugson, B., Penner, B., Rodenbeck, J., Harriss, H., ... Milligan, B. (2020). Field Notes on Pandemic Teaching: 1. Places Journal. https://doi.org/10.22269/200414

McGuigan, M. (2020). Transitioning to Online Learning. Retrieved from https://www.csd.org/stories/transitioning-to-online-learning/

Shaughnessy, M. F., \& Fulgham, S. (2011). Pedagogical models: The discipline of online teaching Hauppauge, NY Nova Science publishers.

Shaughnessy, M. F., Johnson, A., Viner, M., Flores, G., \& Singh, A. (2019). Factors contributing to Student Success in Online and Media Site Courses: A Preliminary Study. Journal of Education, Society and Behavioral Science, 32(3), 1-2. https://doi.org/10.9734/jesbs/2019/v32i330175

Welborn, A., \& Gringle, M. (2020). Showing sincere care: Acknowledging the elephant in the room. Lippincott Nursing Education Blog. Retrieved from http://nursingeducation.lww.com/blog.entry.html/2020/05/15/showing_sincere_care-druo.html

Zschar, H., \& Smith, J. A. (2008). Transition from the Classroom to the Web: Successful Strategies for Teaching Online. Nursing Education Perspectives, 29(1), 23-28.

\section{Copyrights}

Copyright for this article is retained by the author(s), with first publication rights granted to the journal.

This is an open-access article distributed under the terms and conditions of the Creative Commons Attribution license (http://creativecommons.org/licenses/by/4.0/). 